Design Prospective general population birth cohort study with data from birth to four years, drawn from the Southampton Women's Survey. Grip strength was measured using a Jamar handgrip dynamometer at age four years. We also measured height and weight and assessed body composition (lean mass) using dual energy x-ray absorptiometry. Multiple regression was used to relate grip strength to birthweight allowing for adjustment for confounding factors.

Setting Southampton, UK.

Participants 968 children took part in a sub-study assessing body composition and had their grip strength measured at age four years. Their socio-demographic characteristics were similar to the remainder of the cohort.

Main outcome measure Grip strength in kilograms.

Results Birthweight was positively associated with grip strength, with each kilogram of birthweight being associated with a $0.5 \mathrm{~kg}$ increase in grip strength (95\% CI 0.30 to 0.70). Grip strength was also strongly related to current body size and adjustment of the birthweight relationship for height and weight attenuated the relationship such that it became non-significant with a $1 \mathrm{~kg}$ increase in birthweight being associated with $0.15 \mathrm{~kg}$ ( $95 \%$ CI -0.05 to $0.34 \mathrm{~kg}$ ) increase in grip strength. Adjustment for absolute and percentage lean mass instead of height and weight attenuated the relationship still further such that only a $0.07 \mathrm{~kg}$ ( $95 \% \mathrm{CI}-0.16$ to 0.30 ) increase in grip strength was seen in relation to a $1 \mathrm{~kg}$ increase in birthweight.

Conclusion Birthweight is associated with muscle strength in children aged 4 years. Adjustment for current body size or lean mass explains the relationship suggesting that muscle size is on the causal pathway. Early influences on muscle growth and development appear to impact on grip strength in children as well as adults.

\section{THE EFFECT OF MISSING DATA ON THE RELATIONSHIP BETWEEN LIFECOURSE SOCIO-ECONOMIC POSITION AND VERBAL COGNITIVE ABILITY AT OLDER AGES}

doi:10.1136/jech.2010.120956.30

${ }^{1} \mathrm{R}$ Landy, ${ }^{1} \mathrm{R}$ Hardy, ${ }^{2} \mathrm{~J}$ Head, ${ }^{1} \mathrm{M}$ Richards. ${ }^{1} \mathrm{MRC}$ Unit for Lifelong Health and Ageing, University College London, UK; ${ }^{2}$ Department of Epidemiology and Public Health, University College London, UK

Objective To compare the effects of accounting for different missing data mechanisms in an investigation of the role of lifecourse socioeconomic position (SEP) on later-life verbal cognitive ability.

Design Two UK prospective cohort studies.

Participants A nationally representative sample born in 1946 (NSHD) (original $\mathrm{N}=5362$ ), and a sample of British civil servants (Whitehall II) (original $\mathrm{N}=10308$ ).

Methods Linear regression models were used to test associations between SEP at different life stages and verbal ability. Results from complete case analysis (assuming missing completely at random) were compared with those using multiple imputation (assuming missing at random) and a Heckman selection model (assuming missing not at random) for each cohort.

Main outcome measure Verbal cognitive ability in adulthood; the National Adult Reading Test at age 53 years (NSHD), and the Mill Hill Test at ages 55-79 years (Whitehall II).

Results NSHD: Educational qualifications and head of household occupational SEP at age 53 were significantly related to verbal ability using all missing data methods, after adjusting for sex and cognitive function at age 8 . The effect of childhood SEP was not significant at the $5 \%$ level when using Heckman selection (regression coefficient 0.51 ( $95 \%$ CI -0.25 to 1.27$)$ ) but was significant for complete case analysis (regression coefficient 0.83 (95\% CI 0.11 to 1.54)). Compared with complete case analysis, the coefficients for SEP were generally higher when multiple imputation was used, but the overall conclusions remained the same. The coefficients using Heckman selection differed from those for the complete case and multiple imputation analyses, with lower coefficients for all SEP variables. Whitehall II: Educational qualifications and current occupational SEP were significantly associated with verbal ability for all missing data methods, after adjusting for age, sex, marital status, employment status (working/retired/long-term sick) and number of times the cognitive tests had been taken. The effect of childhood SEP was not significant. The coefficients were generally higher for multiple imputation than complete case analysis, whereas the Heckman selection coefficients were lower for educational qualifications and adult SEP (regression coefficient (95\% CI) -4.46 $(-5.12$ to -3.78$)$ for Heckman selection vs -5.15 ( -5.75 to -4.55$)$ for complete case).

Conclusion Educational qualifications and adult SEP were significant predictors of verbal ability in middle to older age, but results for childhood SEP were inconclusive. Greater differences exist between the results from different missing data methods in the older Whitehall II sample, which may be due to greater selective dropout, which is better accounted for by Heckman selection.

\section{Socio-economic status and cardiovascular risk 031 SOCIO-ECONOMIC PATTERNING OF THE TRENDS IN CARDIOVASCULAR DISEASE RISK FACTORS IN SCOTLAND: SCOTTISH HEALTH SURVEYS 1995 TO 2008}

doi:10.1136/jech.2010.120956.31

${ }^{1} \mathrm{~J}$ W Hotchkiss, ${ }^{1} \mathrm{C}$ A Davies, ${ }^{1} \mathrm{~L}$ Gray, ${ }^{2} \mathrm{C}$ Bromley, ${ }^{3} \mathrm{~S}$ Capewell, ${ }^{1} \mathrm{~A}$ H Leyland. ${ }^{1} \mathrm{MRC}$ CSO Social and Public Health Sciences Unit, Glasgow, UK; ${ }^{2}$ Scottish Centre for Social Research, Edinburgh, UK; ${ }^{3}$ Division of Public Health, University of Liverpool, Liverpool, UK

Background In Scotland, as in other countries, cardiovascular disease (CVD) mortality has substantially declined over time. However, this decline may be slowing among younger groups and there are still large inequalities in mortality between socio-economic groups.

Objectives To examine secular changes in the prevalence of known CVD risk factors in the Scottish population according to socioeconomic position.

Design Representative data on health and health-related behaviours from the Scottish Health Surveys, collected by stratified and clustered probability sampling

Participants Data are available from four surveys: 1995, 1998, 2003 and 2008 (6190, 6656, 5497 and 4202 respondents respectively aged 25-64 years).

Main outcome measures Prevalence of cigarette smoking, excess alcohol consumption (men $>21$, women $>14$ units/week), hypertension (self-reported), diabetes (self-reported) and obesity (measured $\mathrm{BMI} \geq 30 \mathrm{~kg} / \mathrm{m}^{2}$ ).

Methods Prevalences, adjusted for survey weights, and stratified by gender and by gender/age were determined according to the individual's social class (Registrar General's) and educational level (highest qualification), standardised to the European standard population. Time-trends and trends across socio-economic categories were assessed using linear regression. The slope index of inequality (SII) was calculated for each risk factor in every survey. Results In 1995 the prevalence of smoking among professionals was $14.1 \%$ (95\% CI 9.5 to $18.8 \%$ ) compared to unskilled workers $51.0 \%$ (46.1 to $55.8 \%$ ) with SII of 39.0. By 2008 the SII for smoking had decreased slightly to 35.7 , with decreased prevalence in all social classes, except in the unskilled. Self-reported hypertension increased moderately within most educational levels; in men the corresponding SII changed little between the first and last survey (8.8 and 8.2 respectively) while for women it increased from 4.2 to 12.1 . Diabetes prevalence increased in all educational categories. By 2008, the 